\title{
MÉTODO GENERALIZADO DE ANÁLISE DE DIALELOS DESBALANCEADOS ${ }^{1}$
}

\author{
SÍLVIA AGRIPINA GONÇALVES E SILVA², ORLANDO PEIXOTO DE MORAIS ${ }^{3}$, CARLOS AGUSTIN RAVA ${ }^{4}$ \\ e JOAQUIM GERALDO CÁPRIO DA COSTA4
}

\begin{abstract}
RESUMO - Apresenta-se um procedimento generalizado de estimação das capacidades geral e específica de combinação em cruzamentos dialélicos, com número desigual de repetições para tratamentos (genitores e combinações híbridas $\mathrm{F}_{1}$ ), avaliados em um delineamento com restrições na casualização. Neste caso, as médias ajustadas estimadas são interdependentes e heterocedásticas, devendo-se, para estimar os parâmetros desejados, utilizar o modelo linear generalizado de Gauss-Markov. O objetivo deste trabalho foi a dedução teórica do método e sua aplicação a um exemplo prático. Foram analisados os dados obtidos de um dialelo completo, sem os recíprocos, envolvendo cinco genitores: CB 511687-1, CB 733753, Diamante Negro, Rosinha G-2 e Compuesto Chimaltenango 2. Os genitores CB 733753, CB 511687-1 e Diamante Negro contribuem geneticamente para a resistência, enquanto Rosinha G-2 e Compuesto Chimaltenango 2 contribuem para a suscetibilidade do feijoeiro ao crestamento-bacteriano comum. Na maioria dos cruzamentos analisados constatou-se a dominância parcial da suscetibilidade do feijoeiro a Xanthomonas axonopodis pv. phaseoli.
\end{abstract}

Termos para indexação: Phaseolus vulgaris, genitores, híbridos, capacidade combinatória, genética quantitativa, resistência a doenças, métodos de melhoramento, crestamento bacteriano, Xanthomonas axonopodis.

\section{GENERALIZED METHOD FOR ANALYSIS OF UNBALANCED DIALLELS}

\begin{abstract}
A general procedure for estimation of general and specific combining ability in diallelic crosses with unequal number of replications for treatments (parents and $F_{1}$ hybrid combinations), evaluated in a design with restricted randomization, is presented. In this case, the estimates of adjusted means are interdependent and heterocedastic, demanding the use of the Gauss-Marcov generalized linear model. The objective of this study was the theoretical deduction of the model and its application to a practical example. A complete diallel crossing without reciprocals was performed, including five parents: CB 511687-1, CB 733753, Diamante Negro, Rosinha G-2 and Compuesto Chimaltenango 2. The parents CB 511687-1, CB 733753 and Diamante Negro contribute genetically for resistance while Rosinha G-2 and Compuesto Chimaltenango 2 contribute for susceptibility of dry bean to common bacterial blight. Most of the crosses analyzed showed partial dominance for susceptibility of bean to Xanthomonas axonopodis pv. phaseoli.
\end{abstract}

Index terms: Phaseolus vulgaris, parents, hybrids, combining ability, quantitative genetics, pest resistance, breeding methods, bacterial blight, Xanthomonas axonopodis.

\footnotetext{
${ }^{1}$ Aceito para publicação em 17 de janeiro de 2000.

Extraído da dissertação apresentada à Universidade Federal de Goiás (UFG), pelo primeiro autor, para obtenção do título de Mestre.

${ }^{2}$ Eng. Agrôn., M.Sc., Rua R-16, n. 326, Setor Oeste, CEP 74125-170 Goiânia, GO.

${ }^{3}$ Eng. Agrôn., Dr., Embrapa-Centro Nacional de Pesquisa de Arroz e Feijão (CNPAF), Caixa Postal 179, CEP 75375-000 Santo Antônio de Goiás, GO

E-mail: peixoto@cnpaf.embrapa.br, rava@cnpaf.embrapa.br, caprio@cnpaf.embrapa.br

${ }^{4}$ Eng. Agrôn., Dr., Embrapa-CNPAF. Bolsista do CNPq.
}

\section{INTRODUÇÃO}

Nos programas de melhoramento genético a escolha dos genitores para o desenvolvimento de populações, capazes de propiciarem os ganhos de seleção desejados, constitui uma das etapas mais críticas. Nesta escolha, o melhorista dispõe de algumas técnicas, destacando-se entre elas os cruzamentos dialélicos, que permitem a identificação de genitores baseada em seus próprios valores genéticos e, principalmente, nas suas capacidades de se combinarem 
em híbridos que produzem populações segregantes promissoras (Ramalho et al., 1993). Um sistema de cruzamentos dialélicos corresponde ao intercruzamento de $\mathrm{p}$ genitores, dois a dois, produzindo $\mathrm{p}(\mathrm{p}-1) / 2$ híbridos, no caso dos dialelos completos, sem os cruzamentos recíprocos.

Atualmente, encontram-se disponíveis vários métodos de análise dos cruzamentos dialélicos, como os pioneiros de Jinks \& Hayman (1953), Hayman (1954), Griffing (1956) e Gardner \& Eberhart (1966), envolvendo os dialelos balanceados, e os que abordam dialelos parciais, incluindo os circulantes (Kempthorne \& Curnow, 1961), dialelos incompletos e dialelos desbalanceados (Cruz \& Regazzi, 1994). As metodologias de análise dialélica permitem obter estimativas de parâmetros genéticos úteis na seleção dos genitores para hibridação e no entendimento da natureza e magnitude dos efeitos genéticos envolvidos na determinação dos caracteres (Cruz, 1997).

Todas as metodologias de análise dialélica já disponíveis pressupõem a homogeneidade e a independência dos erros relativos às médias, exceto a que trata dos dialelos desbalanceados de Cruz \& Regazzi (1994), que contempla a heterocedasticidade das médias, advinda de número desigual de observações das diferentes combinações híbridas, avaliadas em delineamento experimental inteiramente casualizado. Há situações, contudo, em que as médias, além de se originarem de diferentes números de observações e, portanto, heterocedásticas, podem ter sido obtidas de experimentos com restrições na casualização, sendo, adicionalmente, interdependentes, ou seja, nãoortogonais. Nesses casos especiais devem-se adotar métodos generalizados na estimação dos parâmetros genéticos.

O objetivo deste trabalho foi a dedução teórica de um procedimento de análise de cruzamentos dialélicos, avaliados em delineamento de blocos ao acaso, mas com diferente número de repetições por tratamento (desbalanceado), situação em que as médias são, pelo menos parcialmente, heterocedásticas e não-ortogonais.

\section{MATERIAL E MÉTODOS}

Seja um experimento de avaliação de p genitores e seus $\mathrm{p}(\mathrm{p}-1) / 2$ híbridos na geração $\mathrm{F}_{1}$, utilizando um delinea- mento experimental de blocos incompletos não-balanceados, ou seja, com número desigual de repetições por tratamento. O modelo linear correspondente pode ser caracterizado por:

$\mathrm{Y}_{\mathrm{ij}}=\mathrm{m}+\mathrm{B}_{\mathrm{j}}+\mathrm{T}_{\mathrm{i}}+\varepsilon_{\mathrm{ij}}$

onde:

$\mathrm{Y}_{\mathrm{ij}}$ : observação relativa ao tratamento i no bloco $\mathrm{j}$; $\mathrm{m}$ : constante associada à observação- $Y_{\mathrm{ij}} ; \mathrm{Bj}$ : efeito relativo ao bloco $\mathrm{j} ; \mathrm{T}_{\mathrm{i}}$ : efeito do i-ésimo tratamento; $\varepsilon_{\mathrm{ij}}$ : erro associado à observação $\mathrm{Y}_{\mathrm{ij}}$.

A análise de variância pode ser implementada utilizando-se um procedimento compatível com a estrutura do delineamento em blocos incompletos e que, além de fornecer o vetor $\hat{Y}$ de médias de tratamento ajustadas para efeito de blocos, permita a estimação da matriz de covariância $(\hat{V}(\hat{Y}))$ dessas médias. Detalhes sobre a obtenção de $\hat{V}(\hat{Y})$ podem ser vistos em Breseghello et al. (1998).

\section{Obtenção das estimativas da capacidade geral de com- binação (CGC) e da capacidade específica de combi- nação (CEC) dos genitores}

Em busca de simplificação, os estimadores das médias obtidas (componentes do vetor $\hat{Y}$ ) podem ser representados por $\hat{y}_{\text {ii }}$ e $\hat{y}_{\text {ii }}$, quando correspondem ao genitor i ou ao híbrido $F_{1}$ do genitor i com o genitor i', respectivamente. Em seguida, com base em Griffing (1956), essas médias são expressas em função dos seus efeitos da CGC ( $g_{i}$ e $\left.g_{i}{ }^{\prime}\right)$ e da CEC $\left(\mathrm{s}_{\mathrm{ii}}\right)$, da seguinte forma:

$y_{\mathrm{ii}}=\mu+2 \mathrm{~g}_{\mathrm{i}}+\mathrm{s}_{\mathrm{ii}}+\mathrm{e}_{\mathrm{ii}}$, relativa ao genitor $\mathrm{i}$;

$y_{\text {ii' }}=\mu+\mathrm{g}_{\mathrm{i}}+\mathrm{g}_{\mathrm{i}^{\prime}}+\mathrm{s}_{\mathrm{i}{ }^{\prime}}+\mathrm{e}_{\mathrm{ii}}$, relativa ao híbrido $\mathrm{F}_{1}$ dos genitores i e i', em que:

$\mu$ : constante associada à observação $\mathrm{y}_{\mathrm{ii}}$ ou $\mathrm{y}_{\mathrm{ii}}$;

$\mathrm{e}_{\mathrm{ii}}, \mathrm{e}_{\mathrm{ii}}$ : erro relativo às observações $\mathrm{y}_{\mathrm{ii}} \mathrm{e} \mathrm{y}_{\mathrm{ii}}$, respectivamente.

Matricialmente, estas expressões podem ser denotadas por:

$\mathrm{Y}_{\mathrm{d}}=\mathrm{X}_{\mathrm{d}} \beta+\varepsilon$, que, na sua forma expandida, adquire a seguinte caracterização:

$\left[\begin{array}{l}\mathrm{y}_{11} \\ \mathrm{y}_{12} \\ \vdots \\ \mathrm{Y}_{\mathrm{pp}}\end{array}\right]=\left[\begin{array}{ccccccccc}1 & 2 & 0 & \cdots & 0 & 1 & 0 & \cdots & 0 \\ 1 & 1 & 1 & \cdots & 0 & 0 & 1 & \cdots & 0 \\ \vdots & \vdots & \vdots & \vdots & \vdots & \vdots & \vdots & \vdots & \vdots \\ 1 & 0 & 0 & \cdots & 2 & 0 & 0 & \cdots & 1\end{array}\right]\left[\begin{array}{c}\mu \\ \mathrm{g}_{1} \\ \mathrm{~g}_{2} \\ \vdots \\ \mathrm{g}_{\mathrm{p}} \\ \mathrm{S}_{11} \\ \mathrm{~S}_{12} \\ \vdots \\ \mathrm{S}_{p 2}\end{array}\right]+\left[\begin{array}{c}\varepsilon_{11} \\ \varepsilon_{12} \\ \vdots \\ \varepsilon_{p p}\end{array}\right]$

O subscrito d para Y e X foi adotado para simbolizar que se trata de um modelo dialélico e não de delineamento experimental. 
Como os elementos do vetor $Y_{d}$ são as médias de tratamento, ajustadas para o efeito de bloco, em função dos tratamentos comuns entre blocos, seus respectivos erros (componentes de $\varepsilon$ ) não são homogêneos nem independentes entre si. Conseqüentemente, não é possível adotar diretamente os procedimentos convencionais de análise dialélica, estabelecidos por Griffing (1956), que pressupõem a homogeneidade e a independência dos erros relativos às médias, ou seja, admite-se que $\varepsilon \sim \mathrm{N}\left(\varnothing, \mathrm{I}_{\mathrm{e}}{ }^{2}\right)$. Não é também adequado empregar a metodologia de análise de dialelo desbalanceado, apresentada detalhadamente por Cruz \& Regazzi (1994), porque não contempla uma situação de ortogonalidade das observações disponíveis. Pressupõe-se, na metodologia desse autores uma heterogeneidade das médias da tabela dialélica, mas sem covariâncias entre as mesmas.

Na situação em discussão, o sistema de equações normais adequado é o correspondente ao modelo linear generalizado de Gauss-Markov (Searle, 1971), ou seja:

$\mathrm{X}_{\mathrm{d}} \mathrm{V}_{\mathrm{d}}{ }^{-1} \mathrm{X}_{\mathrm{d}} \beta^{\circ}=\mathrm{X}_{\mathrm{d}} \mathrm{V}_{\mathrm{d}}{ }^{-1} \mathrm{Y}_{\mathrm{d}}$, em que:

$\mathrm{V}_{\mathrm{d}}$ : matriz de covariância das médias ajustadas dos genitores e de seus híbridos. Expressão simplificada de $\hat{V}(\hat{Y})$.

\section{Solução do sistema de equação $\mathbf{X}_{\mathbf{d}} \mathbf{V}_{\mathrm{d}}{ }^{-1} \mathbf{X}_{\mathbf{d}} \beta^{\circ}=\mathbf{X}_{\mathbf{d}} \mathbf{V}_{\mathbf{d}}{ }^{-1} \mathbf{Y}_{\mathrm{d}}$}

O sistema, tal como se apresenta, é indeterminado, porque $\mathrm{X}^{\prime}{ }_{\mathrm{d}} \mathrm{V}_{\mathrm{d}}{ }^{-1} \mathrm{X}_{\mathrm{d}}$ é singular. Para contornar esta situação, deve-se, como nos métodos convencionais de análise dialélica, impor cortes no espaço das soluções, pela adoção de restrições lógicas e convenientes. Para facilitar a compreensão do estabelecimento dessas restrições, considerem-se as transformações matriciais que normalmente são implementadas no modelo linear generalizado $\mathrm{Y}_{\mathrm{d}}=\mathrm{X}_{\mathrm{d}} \beta+\varepsilon$ para a obtenção do sistema de equações normais $\mathrm{X}_{\mathrm{d}}{ }_{\mathrm{d}} \mathrm{V}_{\mathrm{d}}{ }^{-1} \mathrm{X}_{\mathrm{d}} \beta^{\circ}=\mathrm{X}^{\prime}{ }_{\mathrm{d}} \mathrm{V}_{\mathrm{d}}{ }^{-1} \mathrm{Y}_{\mathrm{d}}$.

Para tanto, seja a matriz $\mathrm{F}$, que pode ser obtida pela diagonalização por congruência de $\mathrm{V}_{\mathrm{d}}^{-1}$ (Cruz \& Regazzi, 1994), tal que FF' $=V_{d}^{-1}$. Pré-multiplicando todos os termos da equação $\mathrm{Y}_{\mathrm{d}}=\mathrm{X}_{\mathrm{d}} \beta+\varepsilon$ por $\mathrm{F}^{\prime}$, obtém-se um novo modelo $\mathrm{z}=\mathrm{M} \beta+\delta$, em que os componentes de $\delta$ são distribuídos independentemente, com média nula e variância comum $\sigma_{\delta}^{2}=1$, ou seja, $\delta \sim \mathrm{N}(\varnothing, \mathrm{I})$, sendo $\varnothing$ um vetor nulo e I uma matriz identidade.

Como a matriz $\mathrm{X}_{\mathrm{d}}$ não é de posto coluna completo, $\mathrm{M}$, que é igual a $F^{\prime} X_{d}$, também não o será. A matriz $M$ tem $[\mathrm{p}(\mathrm{p}+1) / 2]+1$ colunas, mas tem posto igual a $\mathrm{p}(\mathrm{p}-1) / 2$. Para tanto, devem ser acrescentadas à matriz $\mathrm{M}, \mathrm{p}+1 \mathrm{li}-$ nhas linearmente independentes e não pertencentes ao espaço linha de $\mathrm{M}$, sob as seguintes restrições, como no método 2 de Griffing (1956):

$$
\begin{aligned}
& \sum_{i=1}^{p} \hat{g}_{i}=0 \text { (uma restrição), } \\
& \hat{s}_{i i}+\sum_{i^{\prime}=1}^{p} \hat{s}_{i i^{\prime}}=0 \text { (p restrições). }
\end{aligned}
$$

Para facilitar a compreensão das consequiências da adoção dessas restrições, sejam as p+1 linhas, acrescentadas a M, identificadas por uma submatriz A. O novo modelo adquire a seguinte configuração:

$$
\left[\begin{array}{l}
\mathrm{Z} \\
\cdots \\
\varnothing
\end{array}\right]=\left[\begin{array}{l}
\mathrm{M} \\
\cdots \\
\mathrm{A}
\end{array}\right] \beta+\left[\begin{array}{l}
\delta \\
\cdots \\
\varnothing
\end{array}\right]
$$

O respectivo sistema de equações normais passa a ser: [M'M + A'A] $\hat{\beta}=\mathrm{M}^{\prime} z$, o que leva à seguinte solução (única, sob as restrições impostas):

$\hat{\beta}=\left(\mathrm{M}^{\prime} \mathrm{M}+\mathrm{A}^{\prime} \mathrm{A}\right)^{-1} \mathrm{M}^{\prime} \mathrm{z}$.

Sendo, contudo, $M=F^{\prime} X_{d}, z=F^{\prime} Y_{d}$ e FF' $=V_{d^{-1}}$, tem-se finalmente:

$\hat{\beta}=\left(X^{\prime}{ }_{d} V_{d}^{-1} X_{d}+A^{\prime} A\right)^{-1} X^{\prime}{ }_{d} V_{d}^{-1} Y_{d}$

\section{Matriz de covariância de $\hat{\beta}$}

Para se avaliar a precisão das estimativas dos parâmetros, bem como comparar os contrastes entre estas mesmas estimativas, obtém-se a matriz de covariância de $\hat{\beta}$, dada por: $\hat{V}(\hat{\beta})=\left(M^{\prime} M+A^{\prime} A\right)^{-1} M^{\prime} M\left(M^{\prime} M+A^{\prime} A\right)^{-1}$ ou $\hat{V}(\hat{\beta})=\left(X^{\prime}{ }_{d} V_{d}{ }^{-1} X_{d}+A^{\prime} A\right)^{-1} X^{\prime}{ }_{d} V_{d}{ }^{-1} X_{d}\left(X^{\prime}{ }_{d} V_{d}{ }^{-1} X_{d}+\right.$ $\left.\mathrm{A}^{\prime} \mathrm{A}\right)^{-1}$

\section{Um exemplo prático}

Foi realizado um dialelo completo, sem os recíprocos, envolvendo cinco genótipos de feijoeiro comum, sendo três resistentes ao crestamento bacteriano comum (CB 511687-1, CB 733753 e Diamante Negro) e dois suscetíveis (Rosinha G-2 e Compuesto Chimaltenango 2). A geração $F_{1}$ desses cruzamentos e seus genitores foram submetidos ao ataque dessa doença em condições de casa de vegetação.

De cada cruzamento, plantas dos genitores e da primeira geração $\left(F_{1}\right)$ receberam inóculo mediante incisão das folhas primárias, com uma tesoura previamente mergulhada em uma suspensão de $5 \times 10^{7} \mathrm{ufc} / \mathrm{mL}$ do isolado Xp CNF 15 (Rava, 1984) de Xanthomonas axonopodis pv. phaseoli. Nos cruzamentos em que Rosinha G-2 não participou como genitor, foram incluídas 20 plantas dessa 
cultivar, para definir, em razão da sua maior suscetibilidade, a época mais adequada de avaliação dos sintomas.

A avaliação dos sintomas foi realizada de nove a dez dias após a inoculação, em virtude da temperatura ambiente, quando as plantas da cultivar Rosinha G-2 apresentavam uma média de severidade de doença em torno de 5, conforme escala de notas estabelecida por Rava (1984). Pela inviabilidade de se efetuar a inoculação e a posterior avaliação de todos os tratamentos em uma mesma época, decidiu-se fazê-los por etapa, ou seja, um cruzamento, juntamente com seus respectivos genitores, por vez. Desta forma, cada cruzamento foi submetido à avaliação em época distinta das dos demais. Além da cultivar Rosinha G-2, que foi utilizada em todas as épocas de avaliação como tratamento-controle, ou como genitor, havia outros tratamentos comuns entre épocas de avaliação, representado pelos genitores que participavam, cada um deles, de quatro cruzamentos e, por conseguinte, de quatro épocas.

Tomando época como bloco, tem-se, neste exemplo, uma condição típica de experimento em blocos incompletos, podendo a estimação dos parâmetros de capacidade de combinação ser realizada como apresentado anteriormente.

O número de plantas com inoculação e avaliadas era variável (20 a 50) de uma parcela para outra. Decidiu-se, por isso, efetuar a análise de variância ao nível de plantas, utilizando o modelo estatístico anteriormente exposto, tendo, contudo, a soma de quadrados da variação entre plantas dentro de parcelas e os respectivos graus de liberdade sido posteriormente excluídos da soma de quadrados e graus de liberdade residuais, obtendo-se o erro entre parcelas, com apenas 12 graus de liberdade. A análise de variância foi realizada utilizando-se o procedimento PROC GLM do módulo STAT do sistema SAS (SAS Institute, 1993).

\section{RESULTADOS E DISCUSSÃO}

As estimativas de nota média de reação foliar ao crestamento-bacteriano comum, ajustadas para efeito de épocas de avaliação, dos híbridos e seus respectivos genitores, bem como a matriz de covariância dessas estimativas, estão apresentadas na Tabela 1. Não se observou uma interdependência forte e generalizada entre as médias estimadas, mas, principalmente quando se comparam os cruzamentos com seus genitores, a covariação pode atingir níveis relevantes. Cita-se, como exemplo, o caso da estimativa de covariância entre a média da $F_{1}$ do cruzamento do genitor 1 com o genitor $2, \mathrm{~F}_{1}(12)$, e a do genitor 1 
(G1), que corresponde a $36,6 \%$ da estimativa de variância deste genitor. Decidiu-se pela apresentação dessa matriz, principalmente para fornecer elementos para exercício dos principais passos do método.

Na Tabela 2, apresenta-se a matriz de covariância das estimativas de capacidade geral de combinação $(\hat{g})$, que constitui parte de $\hat{\mathrm{V}}(\hat{\beta})$. Ela permite avaliar se cada estimativa de $\mathrm{g}$ difere significativamente de zero (teste t), bem como se há diferenças significativas entre os genitores quanto à capacidade geral de combinação, considerando que a variância de um dado contraste, $\mathrm{y}=\hat{g}_{\mathrm{i}}-\hat{g}_{\mathrm{i}}$, , contempla também a covariância entre $\hat{g}_{\mathrm{i}} \mathrm{e} \hat{g}_{\mathrm{i}}$ '.

As estimativas dos efeitos da capacidade geral de combinação proporcionam informações sobre a concentração de genes predominantemente aditivos em seus efeitos (Cruz \& Regazzi, 1994). Portanto, genitores com estimativas positivas de $\mathrm{g}_{\mathrm{i}}$ são os que mais contribuem para o aumento da expressão do caráter em questão. Para o caso de avaliação de resistência a doenças, o interesse se concentra nos genótipos com menor severidade de doença, ou seja, genótipos que contribuem para diminuir a expressão do caráter e, conseqüientemente com estimativas negativas de $\mathrm{g}_{\mathrm{i}}$. Observa-se, na Tabela 2, que os genótipos CB 511687-1, CB 733753 e Diamante Negro foram os que apresentaram as melhores capacidades gerais de combinação para resistência a $X$. axonopodis pv. phaseoli, com estimativas de - 0,670 ; $-0,579$; e $-0,481$, respectivamente. Os genótipos Compuesto Chimaltenango 2 e Rosinha G-2 apresentaram estimativas positivas de $\mathrm{g}_{\mathrm{i}}$, o que implica contribuição genética favorável à suscetibilidade.
A capacidade geral de combinação mais desfavorável foi a de Rosinha G-2, sendo, por conseguinte, o genitor que apresentou a menor capacidade para contribuir com alelos de resistência para o conjunto gênico em estudo.

As estimativas dos efeitos da capacidade específica de combinação do genitor i com i' ( $\left.\hat{\boldsymbol{S}}_{\text {ii }}\right)$, participantes do dialelo, e seus desvios-padrão, são apresentados na Tabela 3 .

A estimativa de $\mathrm{s}_{\mathrm{ii}}$ ' relativa ao cruzamento Rosinha G-2 (G2) x Compuesto Chimaltenango 2 (G5) revelou-se negativa e significativa a $5 \%$ pelo teste $\mathrm{t}$. Em conseqüência, este cruzamento comportou-se relativamente melhor que o esperado, considerando as estimativas de $\mathrm{g}_{\mathrm{i}}$ dos seus genitores, que foram positivas e altamente significativas.

Os cruzamentos CB 511687-1 (G1) x Rosinha G-2 (G2), Rosinha G-2 (G2) x CB 733753 (G3) e Diamante Negro (G4) x Compuesto Chimaltenango 2 (G5) apresentaram altas estimativas positivas de $s_{i i}$, (Tabela 3 ). Adicionalmente, as estimativas de $\mathrm{g}_{\mathrm{i}}$ para Rosinha G-2 e Compuesto Chimaltenango 2 foram altas e positivas (Tabela 2), resultando em médias altas para as $\mathrm{F}_{1}$ dos referidos cruzamentos (4,36, 4,49 e 3,92, respectivamente).

A comparação entre as médias de todos os cruzamentos e genitores pode ser feita com auxílio da matriz de contrastes da Tabela 4. Como a variância da diferença entre duas médias depende do número de observações associadas a estas duas médias e da intensidade de interdependência entre as mesmas, dois contrastes quaisquer da referida matriz podem ter duas diferenças mínimas significativas distintas. É por isso que, por exemplo, um contraste de 1,67

TABELA 2. Estimativas de capacidade geral de combinação $(\hat{g})$, para reação foliar à Xanthomonas axonopodis pv. phaseoli e de covariâncias entre as mesmas, para cinco genitores de Phaseolus vulgaris ${ }^{1}$.

\begin{tabular}{lrrrrrrrr}
\hline Genitor (G1) & $\hat{g}_{\mathrm{i}}$ & Teste t & Probabilidade $(\mathrm{p})$ & \multicolumn{4}{c}{ Covariância $\left(10^{-3}\right)$} \\
\cline { 5 - 8 } & & & & $\hat{\mathrm{g}}_{1}$ & $\hat{g}_{2}$ & $\hat{g}_{3}$ & $\hat{g}_{4}$ & $\hat{\mathrm{g}}$ \\
\hline CB 511687-1 (G1) & $-0,670 \mathrm{a}$ & 7,01 & $\mathrm{p}<0,001$ & 9,140 & $-1,575$ & $-2,458$ & $-2,336$ & $-2,772$ \\
Rosinha G-2 (G2) & $1,127 \mathrm{c}$ & 12,71 & $\mathrm{p}<0,001$ & & 7,861 & $-2,534$ & $-2,565$ & $-1,190$ \\
CB 733753 (G3) & $-0,579 \mathrm{a}$ & 6,02 & $\mathrm{p}<0,001$ & & & 9,230 & $-1,964$ & $-2,274$ \\
Diamante Negro (G4) & $-0,481 \mathrm{a}$ & 4,93 & $\mathrm{p}<0,001$ & & & & 9,559 & $-2,693$ \\
C.C.2 (G5) & $0,603 \mathrm{~b}$ & 6,38 & $\mathrm{p}<0,001$ & & & & & 8,926 \\
\hline
\end{tabular}

${ }_{1}^{1}$ Médias seguidas pela mesma letra não apresentam diferenças significativas pelo teste Tukey, a 5\% de probabilidade; C.C.2: Compuesto Chimaltenango 2 ; teste $\mathrm{t}$ com 12 graus de liberdade. 
TABELA 3. Estimativas dos efeitos da capacidade específica de combinação ( $\hat{s}_{\text {iii }}$ ) e os respectivos desvios-padrão, $\mathrm{s}\left(\hat{s}_{\mathrm{ii}}\right)$, para reação foliar a Xanthomonas axonopodis pv. phaseoli, de cinco genitores de Phaseolus vulgaris.

\begin{tabular}{|c|c|c|c|c|}
\hline \multicolumn{2}{|c|}{ Genitor $^{1}$} & \multirow{2}{*}{$\hat{\boldsymbol{S}}_{\mathrm{ii}}$} & \multirow[t]{2}{*}{ Teste $\mathrm{t}$} & \multirow[t]{2}{*}{ Probabilidade $(\mathrm{p})$} \\
\hline $\mathrm{i}$ & i' & & & \\
\hline G1 & G2 & 0,678 & 3,17 & $\mathrm{p}<0,01$ \\
\hline G1 & G3 & $-0,234$ & 1,22 & $p>0,10$ \\
\hline G1 & G4 & $-0,141$ & 0,63 & $\mathrm{p}>0,10$ \\
\hline G1 & G5 & $-0,289$ & 1,66 & $p>0,10$ \\
\hline G2 & G3 & 0,718 & 3,68 & $\mathrm{p}<0,01$ \\
\hline G2 & G4 & $-0,268$ & 1,27 & $p>0,10$ \\
\hline G2 & G5 & $-0,482$ & 2,23 & $\mathrm{p}<0,05$ \\
\hline G3 & G4 & 0,257 & 1,13 & $p>0,10$ \\
\hline G3 & G5 & 0,144 & 0,77 & $p>0,10$ \\
\hline G4 & G5 & 0,577 & 2,95 & $p<0,02$ \\
\hline G1 & G1 & $-0,014$ & 0,08 & $\mathrm{p}>0,10$ \\
\hline G2 & G2 & $-0,646$ & 3,47 & $\mathrm{p}<0,01$ \\
\hline G3 & G3 & $-0,886$ & 4,95 & $\mathrm{p}<0,01$ \\
\hline G4 & G4 & $-0,425$ & 2,24 & $\mathrm{p}<0,05$ \\
\hline G5 & G5 & 0,051 & 0,29 & $\mathrm{p}>0,10$ \\
\hline
\end{tabular}

G1: CB 511687-1; G2: Rosinha G-2; G3: CB 733753; G4: Diamante Negro; G5: Compuesto Chimaltenango 2.

entre as médias de $\mathrm{F}_{1}(14)$ e de $\mathrm{F}_{1}(24)$ não se revelou significativo ( $p>0,05)$, enquanto outro, de magnitude cerca de duas vezes e meia menor $(0,69)$, entre os genitores 1 e 3, atingiu o nível de significância preestabelecido. Ressalta-se que estes dois genitores foram avaliados em quatro épocas, enquanto cada cruzamento foi avaliado em apenas uma determinada época.

Os sinais negativos das estimativas de $\mathrm{s}_{\mathrm{ii}}$, relativas aos genitores individualmente (Tabela 3), indicam a existência de desvios unidirecionais da dominância e, conseqüentemente, a manifestação de heterose positiva nas combinações híbridas, envolvendo os genitores divergentes (Tabela 4). Os $\mathrm{s}_{\mathrm{ii}} \mathrm{de}$ maiores magnitudes (valor absoluto) foram apresentados pelos genitores Rosinha G-2 e CB 733753, fato que evidencia a divergência genética dos mesmos em relação à média dos outros genitores envolvidos no dialelo, bem como o maior efeito da heterose varietal manifestada na $F_{1}$ do cruzamento entre os

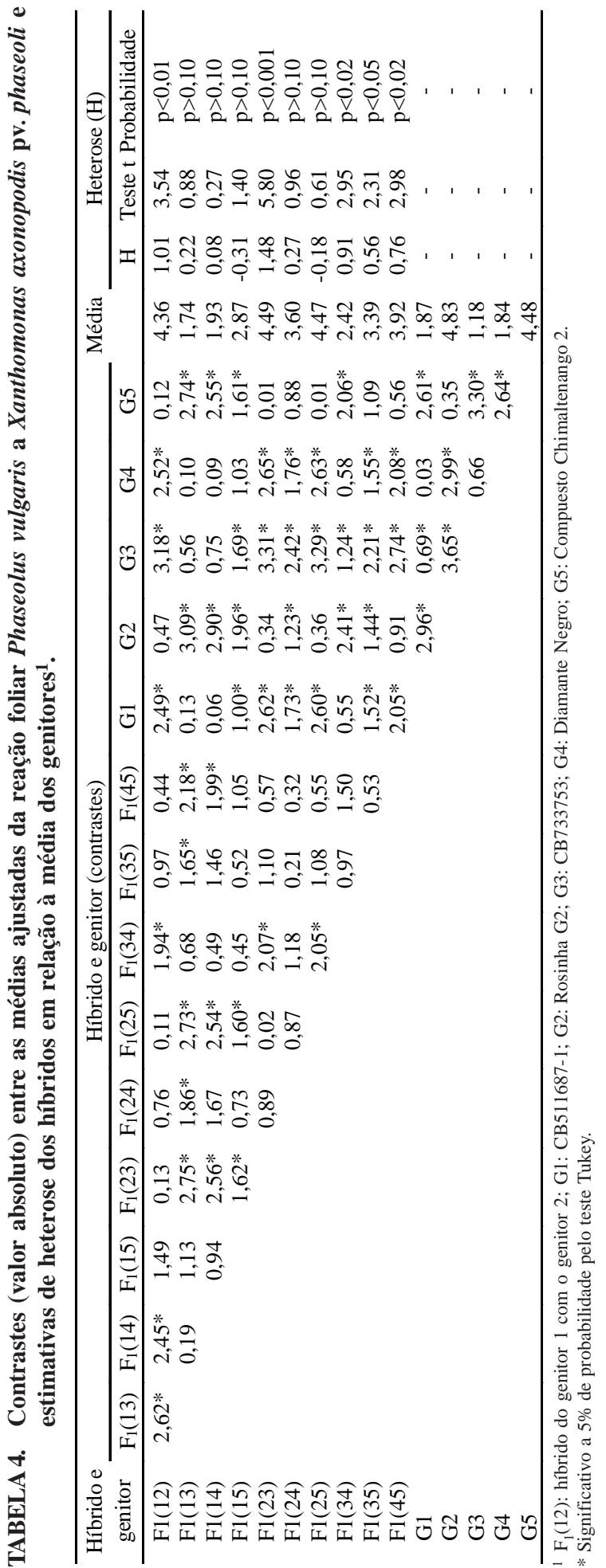


mesmos. Observa-se, ainda, que a maioria das estimativas significativas da heterose são positivas, indicando dominância da suscetibilidade.

\section{CONCLUSÕES}

1. O método generalizado de análise de dialelos desbalanceados permite a análise de dialelos avaliados em experimentos com número diferente de repetições e com restrição na casualização.

2. Os genitores CB 733753, CB 511687-1 e Diamante Negro contribuem geneticamente em forma expressiva para a resistência, enquanto Rosinha G-2 e Compuesto Chimaltenango 2 contribuem para a suscetibilidade do feijoeiro ao crestamento bacteriano comum.

3. Na maioria dos cruzamentos analisados constata-se a dominância parcial da suscetibilidade do feijoeiro a Xanthomonas axonopodi pv. phaseoli.

\section{REFERÊNCIAS}

BRESEGHELLO, F.; MORAIS, O.P.; RANGEL, P.H.N A new method to estimate genetic gain in annual crops. Genetics and Molecular Biology, Ribeirão Preto, v.21, n.4, p.551-555, 1998.

CRUZ, C.D. Programa GENES: aplicativo computacional em genética e estatística. Viçosa : UFV, 1997. 442p.

CRUZ, C.D.; REGAZZI, A.J. Modelos biométricos aplicados ao melhoramento genético. Viçosa : UFV, 1994. 390p.
GARDNER, C.O.; EBERHART, S.A. Analysis and interpretation of the variety cross diallel and related populations. Biometrics, Washington, v.22, p.439452,1966

GRIFFING, B. Concept of general and specific combining ability in relation to diallel crossing systems. Australian Journal of Biological Sciences, Collingwood, v.9, p.463-493, 1956.

HAYMAN, B.I. The theory and analysis of diallel crosses. Genetics, Bethesda, v.39, p.789-909, 1954.

JINKS, J.L.; HAYMAN, B.I. The analysis of diallel crosses. Maize Genetics Cooperation Newsletter, Columbia, v.27, p.48-54, 1953.

KEMPTHORNE, O.; CURNOW, R.N. The partial diallel cross. Biometrics, Washington, v.17, p.229-250, 1961.

RAMALHO, M.A.P.; SANTOS, J.B. dos; ZIMMERMANN, M.J. de P. Genética quantitativa em plantas autógamas: aplicações ao melhoramento do feijoeiro. Goiânia : Editora da UFG, 1993. $271 \mathrm{p}$.

RAVA, C.A. Patogenicidade de isolamentos de Xanthomonas campestris pv. phaseoli. Pesquisa Agropecuária Brasileira, Brasília, v.19, n.4, p.445448, abr. 1984

SAS INSTITUTE (Cary, Estados Unidos). SAS/STAT software: syntax: version 6. Cary, 1993. 151p.

SEARLE, S.R. Linear models. New York : J. Wiley, 1971. $532 \mathrm{p}$. 